\title{
Electroacupuncture Provides a New Approach to Neuroprotection in Rats with Induced Glaucoma
}

\author{
HENRY H.L. CHAN, Ph.D., ${ }^{1}$ MASON C.P. LEUNG, Ph.D., ${ }^{2}$ and KWOK-FAI SO, Ph.D. ${ }^{3}$
}

\begin{abstract}
Objectives: To investigate, using multifocal electroretinogram (mfERG), the effect of electroacupuncture (EA) on retinal function in rats with experimental glaucoma.

Design and subjects: Glaucoma was induced in the right eyes of 15 adult female Sprague-Dawley rats by laser photocoagulation for three quarters of the perilimbal and episcleral vessels. The left eye of each rat was used as the control. The animals were divided into 3 groups: no treatment (non-EA control group), $2 \mathrm{~Hz}$ EA group, and $100 \mathrm{~Hz}$ EA group. EA treatment at different frequencies can produce different levels of analgesia and hence the effect of EA with different frequencies on glaucoma treatment was investigated. Both eyes of each rat in the EA experimental groups received 3 EA treatment sessions each week for 4 weeks. The retinal function was measured using mfERG after 4 weeks of EA treatment.

Results: There was no significant difference in the amplitude (both N1 trough and P1 peak) of mfERG firstorder kernel response between the treatment and control groups. In determining the waveform characteristics by the ratio of $\mathrm{N} 1$ amplitude to $\mathrm{P} 1$ amplitude (N/P ratio), obvious differences were found in the N/P ratio between the control eyes and the glaucoma eyes in the non-EA group and the $100 \mathrm{~Hz}$ EA treatment group, but similar values in the N/P ratio were observed between the control eyes and the glaucoma eyes in the $2 \mathrm{~Hz}$ EA treatment group. The waveform from the eyes with glaucoma was deformed in both the non-EA group and the $100 \mathrm{~Hz}$ EA group, but the waveform from the glaucomatous eye was preserved in the $2 \mathrm{~Hz}$ EA group.

Conclusions: Application of EA at $2 \mathrm{~Hz}$ provides neuroprotection by preserving retinal function in rats with experimental glaucoma. Low frequency EA may be an alternative therapy in the treatment of glaucoma.
\end{abstract}

\section{INTRODUCTION}

G laucoma is the second leading cause of blindness worldwide. It is characterized by optic neuropathy with progressive loss of retinal ganglion cells (RGCs) causing excavation of the optic disc and finally visual field loss (Nickells, 1996; Quigley et al., 1981). There are at least two theories of glaucoma: The mechanical theory suggests that the damage is a result of compression of the axons from elevated intraocular pressure (IOP) at the optic nerve head, while the vascular theory suggests that high IOP induces ischemia by limiting the blood supply to the retina (Anderson, 1987).

While mechanical compression and ischemia may be the initial insults (Schwartz et al., 1996), other primary de- structive factors have also been suggested for the apoptosis of RGCs (Garcia-Valenzuela et al., 1995; Kerrigan et al., 1997; Quigley et al., 1995), such as the short-term release of glutamate (Dreyer et al., 1996) or nitric oxide (NO) (Morgan et al., 1999; Siu et al., 2002), and a shortage of neurotrophic factors.

One of the isoforms of nitric oxide synthase, nitric oxide synthase-2 (NOS-2), also known as induced nitric oxide synthase (iNOS), seems to play an important role in glaucomatous damage (Becquet et al., 1997; Neufeld et al., 1999; Siu et al., 2002). NOS-2 is transiently induced after exposure to cytokines or endotoxins and produces a large quantity of NO, which combines with superoxide to form a peroxynitrite anion. The peroxynitrite anion then decomposes

Departments of ${ }^{1}$ Optometry and Radiography and ${ }^{2}$ Rehabilitation Sciences, Hong Kong Polytechnic University, Hong Kong, China. ${ }^{3}$ Department of Anatomy, University of Hong Kong, Hong Kong, China. 
to form toxic hydroxyl free radical $\left(\mathrm{OH}^{-}\right)$(Lipton et al., 1993) and triggers apoptosis (Garcia-Valenzuela et al., 1995; Kerrigan et al., 1997; Quigley et al., 1995). This new hypothesis of glaucomatous etiology has led to several studies exploring new treatment by inhibiting NOS-2.

Aminoguanidine, an NO inhibitor, has been used by Neufeld and colleagues (1999) to investigate neuroprotection in glaucoma. Their results indicate that the axons at the optic nerve head of rats with glaucoma were protected after administration of the NO inhibitor. The study showed that reduction of NOS-2 can help to lessen glaucomatous damage.

Acupuncture is a branch of Traditional Chinese Medicine (TCM) which has been used for more than 2000 years in the treatment of various diseases (Lin, 1991; Shao and Ding, 1985; Song, 1993; Stux and Pomeranz, 1988). Recent studies suggest that acupuncture causes inhibition of NOS (Leung et al., 2000; Yang et al., 2000). Leung and colleagues (2000) have shown that the total functional activity of NOS decreased significantly in glaucomatous rat eyes after EA treatment. Dabov and colleagues (1985) reported significant decreases in the IOP of glaucoma patients after acupuncture treatment. These studies support the idea that acupuncture could produce neuroprotective effects in glaucoma. However, no study has been done to measure the retinal function when acupuncture is used as a treatment for glaucoma.

In this study, a rat glaucoma model was used to investigate the effects of acupuncture. Experimentally induced glaucoma in the rat has proven to be a useful protocol for glaucoma studies (Siu et al., 2002). The VERIS multifocal electroretinogram (mfERG) system (EDI, San Francisco, CA) was used to provide objective measurement of the glaucomatous changes and retinal function of the animals. Multiple retinal areas were stimulated simultaneously, and responses from different regions of the retina were examined to give a clear indication of central and pericentral electrical responses. Previous studies have used mfERG to measure glaucomatous changes (Chan and Brown, 1999; Chan and Brown, 2000). These findings have suggested that the mfERG system is an effective and reliable tool for measuring retinal changes in pathological conditions. The present study also investigated the effects of 2 frequencies of EA stimulation on glaucoma treatment, as some previous studies had reported that EA at different frequencies produced different levels of analgesia (Han et al., 1999; Huang et al., 2000).

\section{MATERIALS AND METHODS}

\section{Animal preparation}

Fifteen (15) adult female Sprague-Dawley rats weighing 200-300 g were used. The animals were kept in a temperature-controlled $\left(24^{\circ} \mathrm{C}\right)$ room subjected to a 12 hour light/12 hour dark cycle and were provided with adequate food and water. They were handled in strict accordance with the regulations of the animal ethics subcommittee of The Hong Kong Polytechnic University. Glaucoma was induced in the right eye by laser irradiation to three-quarters of the perilimbal region using standard laser equipment (Ultima ${ }^{\circledR} 2000 \mathrm{SE}$ Argon Laser; Coherent, Ontario, Canada). This technique of laser photocoagulation has been proposed in previous studies ( $\mathrm{Ji}$ et al., 2004; Levkovitch-Verbin et al., 2002; Schori et al., 2001; Siu et al, 2002; WoldeMussie et al., 1997; WoldeMussie et al., 2001). Briefly, the laser photocoagulation of the episcleral and limbal drainage vessels in a 270 degree arc around the perilimbal region (excluding the nasal quadrant) produces sufficient outflow blockages. The left eye was not manipulated in any way and was used as control. Laser photocoagulation was done twice, 7 days apart. Our lab has shown that this laser photocoagulation technique induces significant IOP elevation, ganglion cell death, and glaucomatous damage in rats (Siu et al., 2002). The rats were anesthetized for IOP measurement of each eye by TonoPen (Xomed, Jacksonville, FL). Three sets of readings were recorded to obtain a mean value. The IOP of the rats in each group were measured at the first week and the last week of EA treatment (Table 1).

The rats were divided into 3 groups: 5 rats were used as controls without any EA treatment (non-EA group), 5 rats

Table 1. Intraocular Pressure (Mean and Standard Error of the Mean in Glaucomatous and Control Eyes in 3 Groups (A, B, and C)

\begin{tabular}{lccc}
\hline Conducted after EA treatment & $\begin{array}{c}\text { (A) Non-EA } \\
\mathrm{n}=4\end{array}$ & $\begin{array}{c}\text { (B) } 2-\mathrm{Hz} E A \\
\mathrm{n}=5\end{array}$ & $\begin{array}{c}\text { (C) } 100-H z E A \\
\mathrm{n}=5\end{array}$ \\
\hline First week of EA treatment & & & \\
$\quad$ Glaucomatous (right) eye & $28.25 \pm 4.35 \mathrm{mmHg}$ & $20.83 \pm 5.38 \mathrm{mmHg}$ & $19.33 \pm 3.56 \mathrm{mmHg}$ \\
$\quad$ Control (left) eye & $23.33 \pm 6.11 \mathrm{mmHg}$ & $20.83 \pm 2.79 \mathrm{mmHg}$ & $24.0 \pm 3.35 \mathrm{mmHg}$ \\
Last week of EA treatment & $26.75 \pm 2.75 \mathrm{mmHg} *$ & $19.67 \pm 3.33 \mathrm{mmHg}$ & $20.0 \pm 1.41 \mathrm{mmHg} * *$ \\
$\quad$ Glaucomatous (right) eye & $17.50 \pm 3.70 \mathrm{mmHg}$ & $18.33 \pm 2.42 \mathrm{mmHg}$ & $18.5 \pm 1.05 \mathrm{mmHg}$ \\
$\quad$ Control (left) eye & &
\end{tabular}

Note: The intraocular (IOP) pressures in glaucomatous eyes in the nonelectroacupuncture group $(* p<0.05)$ and in the $100-\mathrm{Hz}$ EA group $* * p<0.01)$ were higher than those in the control eyes. The reduction of elevated IOP occurred in glaucomatous eyes after 4 weeks of $2-\mathrm{Hz}$ EA treatment $(p>0.05)$.

EA, electroacupuncture. 
underwent EA treatment at $2 \mathrm{~Hz}$ (2 $\mathrm{Hz}$ EA group), and 5 rats underwent EA at $100 \mathrm{~Hz}(100 \mathrm{~Hz}$ EA group). The EA treatments were started 1 week after the second laser photocoagulation.

\section{EA treatment}

Both treatment groups received EA treatment on days 1, 3 , and 5 each week for 4 weeks. Prior to each EA treatment, the animal received general anesthesia with an intraperitoneal injection of ketamine $10 \%$ ( $0.2 \mathrm{~mL}$ per $300 \mathrm{~g}$ body weight $)$ (Alfamedic) and xylazine $2 \%$ ( $0.1 \mathrm{~mL}$ per $300 \mathrm{~g}$ body weight) (Alfamedic) and, if necessary, a supplemental dose was given after approximately 45 minutes. The rat was then placed on a plastic board and the body covered with a cloth to keep it warm during the EA treatment. Sterile disposable acupuncture needles were inserted bilaterally at acupoints A and B, which correspond to the acupoints HN4 (Extra Point Yuyao) and ST1 (Chengqi) in the human (Fig. 1). The electrical stimulus was generated by an electrostimulator (ITO IC-4107, Japan) with an electrical current of approximately $1 \mathrm{~mA}$ for both $2 \mathrm{~Hz}$ and $100 \mathrm{~Hz}$ EA groups. Each EA treatment lasted 20 minutes and both eyes (control eye and glaucoma eye) received the treatment simultaneously.

\section{mfERG recording}

The mfERG system generated visual stimuli to multiple retinal areas and measured the retinal responses of each of these areas. It analyzed the retinal responses using kernel analysis to assess nonlinear function of the retina (Chan and Brown, 1999; Chan and Brown, 2000). The mfERG recording was performed after 4 weeks of EA treatment. The animal was dark-adapted overnight and anesthetized. Each pupil was dilated with a drop of $1 \%$ tropicamide (Alcon) and the corneal surface was locally anesthetized with a drop

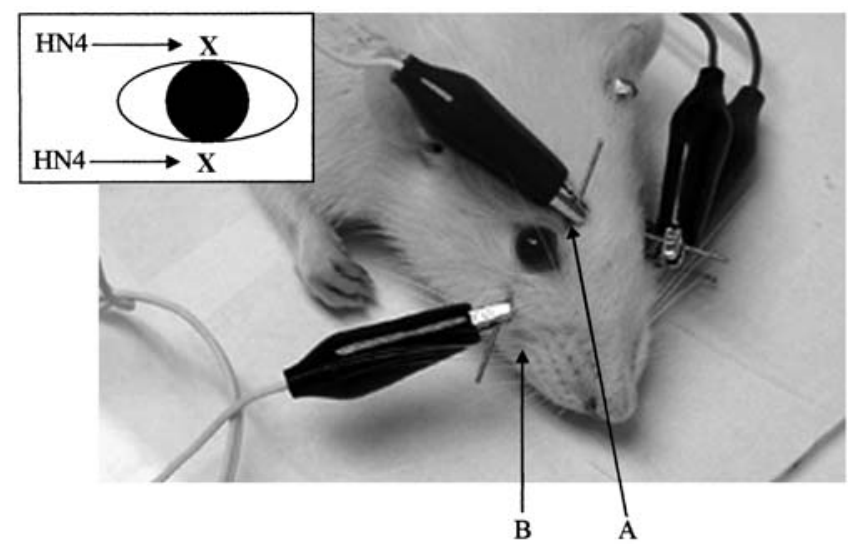

FIG. 1. Needles were inserted in acupoints A and B, which correspond to acupoints HN4 and ST01 in humans (upper left corner). Electrical stimulation was provided by acupunctoscope and the current was fixed at $1 \mathrm{~mA}$. of $0.4 \%$ novesin (Alcon). A silver electrode was placed onto the corneal surface and referenced to a surface electrode on the ipsilateral ear. The ground electrode was placed on the contralateral ear. The preparation and measurement were done in dim red light.

The animal was anesthetized with the same procedure as for the EA treatment, and placed on a movable plastic platform at a height of $16 \mathrm{~cm}$ and positioned $50 \mathrm{~cm}$ from the mfERG stimulus monitor (15-inch Apple RGB monitor). The platform was adjusted so that the line of sight of the rat was aligned with the center of the mfERG stimulus pattern displayed on the monitor. No optical correction was used because the depth of focus for rats is approximately 10 diopters and generally they are emmetropic (Remtulla and Hallett, 1985). After the mfERG recording, the animal was allowed to recover from anesthesia before it was returned to its cage.

\section{Stimulus for multifocal ERG measurement}

A 61-hexagon nonscaled stimulus array was used for the multifocal ERG measurement in this study. The visual stimulus was generated by the Visual Evoked Response Imaging System (VERIS 1.0; EDI, San Francisco) on the stimulus monitor. The stimulus pattern was about $24 \mathrm{~cm}$ horizontal and $17 \mathrm{~cm}$ vertical, giving an angular subtend of 26 degrees $\times 20$ degrees. A neutral density filter (1.0) and a Kodak W47A blue filter $\left(\lambda_{\max }=470 \mathrm{~nm}\right.$, bandwidth $\approx 20 \mathrm{~nm}$ ) were positioned $1 \mathrm{~cm}$ in front of the tested eye. The stimulus luminance was measured using a Topcon BM-5 Luminance Meter with a 0.2 degree field. The luminance of the stimuli, after passing through the two filters, was $0.005 \mathrm{~cd} / \mathrm{m}^{2}$ for the black hexagon and 1.38 $\mathrm{cd} / \mathrm{m}^{2}$ for the white hexagon (mean luminance $=0.69$ $\mathrm{cd} / \mathrm{m}^{2}, 99 \%$ contrast).

As rats are nocturnal animals, dark blank frames between the stimulus frames were introduced for the recovery of the rod system. Nusinowitz and colleagues (1999) used 14 dark blank frames in between the flashes for the mfERG measurement (the interval is about $14 \times 13.33 \mathrm{msec}=186.6$ $\mathrm{msec})$ in rats. Since the frame rate $(60 \mathrm{~Hz})$ in this study was slower than the one used in their study, the number of inserted blank frames between flashes was 12 and the interval was about $200 \mathrm{msec}(12 \times 16.67 \mathrm{msec})$. This ensured sufficient time for recovery of the rod system. The selected m-sequence was $2^{12}-1$ and required about 14 minutes to complete one recording. Each recording was done in two segments to allow for rechecking of the status and positioning of the animal. Therefore, the total duration for mfERG recording for both eyes was about 30 minutes.

\section{Data analy $\backslash$ sis}

The first-order kernel mfERG response was extracted and averaged to show the variation of the retinal functions 
in different groups. The response of two components (N1 trough and P1 peak) was measured in the experiment (Fig. 2). Apart from the magnitudes of $\mathrm{N} 1$ and $\mathrm{P} 1$, the ratio of $\mathrm{N} 1$ to $\mathrm{P} 1$ (N/P ratio) was calculated to demonstrate the variation of waveform characteristics. Student's paired $t$-test was chosen as the statistical method of data analysis between the eyes in each treatment group, and the multiple $t$ test was chosen to analyze the treatment effect between different groups. The alpha level was 0.05 for the statistical analysis.

\section{RESULTS}

The first-order kernel responses in mfERG of the animals were measured. One rat was excluded from the control group during the first week due to unexpected hypersensitivity to the anesthetic, leaving a total of 14 rats for the mfERG study after 4 weeks of EA treatment. A typical summed 61 mfERG response waveform recorded from the left eye (control eye) of a rat from the control (non-EA) group is shown in Figure 3A. This clearly demonstrates that the control eye has similar waveforms across the retina. Another typical summed 61 mfERG response recorded from the glaucomatous right eye of the same rat is shown in Figure 3B. Changes in the waveforms are seen across the retina, which might reflect functional change following the induced glaucomatous damage.

\section{mfERG response amplitude}

In the non-EA group, relatively large abnormal mfERG responses (both $\mathrm{N} 1$ and $\mathrm{P} 1$ ) were recorded from the glaucomatous eye (Fig. 4). The N1 and P1 responses from both the glaucomatous (right) and control (left) eyes in the $2 \mathrm{~Hz}$ and $100 \mathrm{~Hz}$ EA groups were similar. There was no statistically significant difference in the $\mathrm{N} 1$ and $\mathrm{P} 1$ responses between the two eyes in the control group (N1, $t=1.85, p>$ $0.05 ; \mathrm{P} 1, t=1.02, p>0.05)$ or in either EA treatment groups (2 Hz EA treatment: $\mathrm{N} 1, t=-0.54, p>0.05 ; \mathrm{P} 1$, $t=0.31, p>0.05 ; 100 \mathrm{~Hz}$ EA treatment: N1, $t=-0.30$,

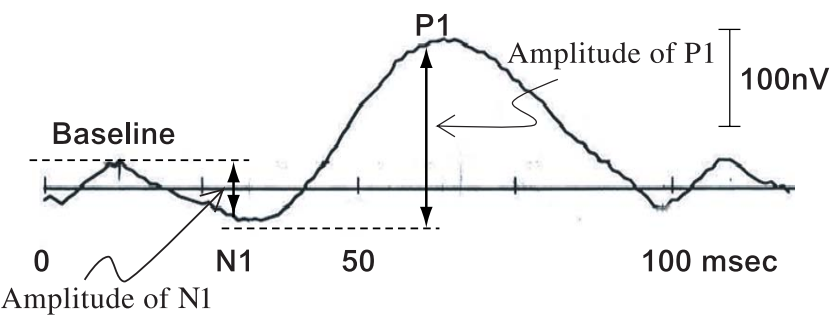

FIG. 2. The multifocal electroretinogram response from the left eye of a rat from the non-electroacupuncture (EA) group. Amplitudes of $\mathrm{N} 1$ and P1 were measured from the baseline to the peak of N1 and the amplitude of P1 was measured from N1 to peak of P1.

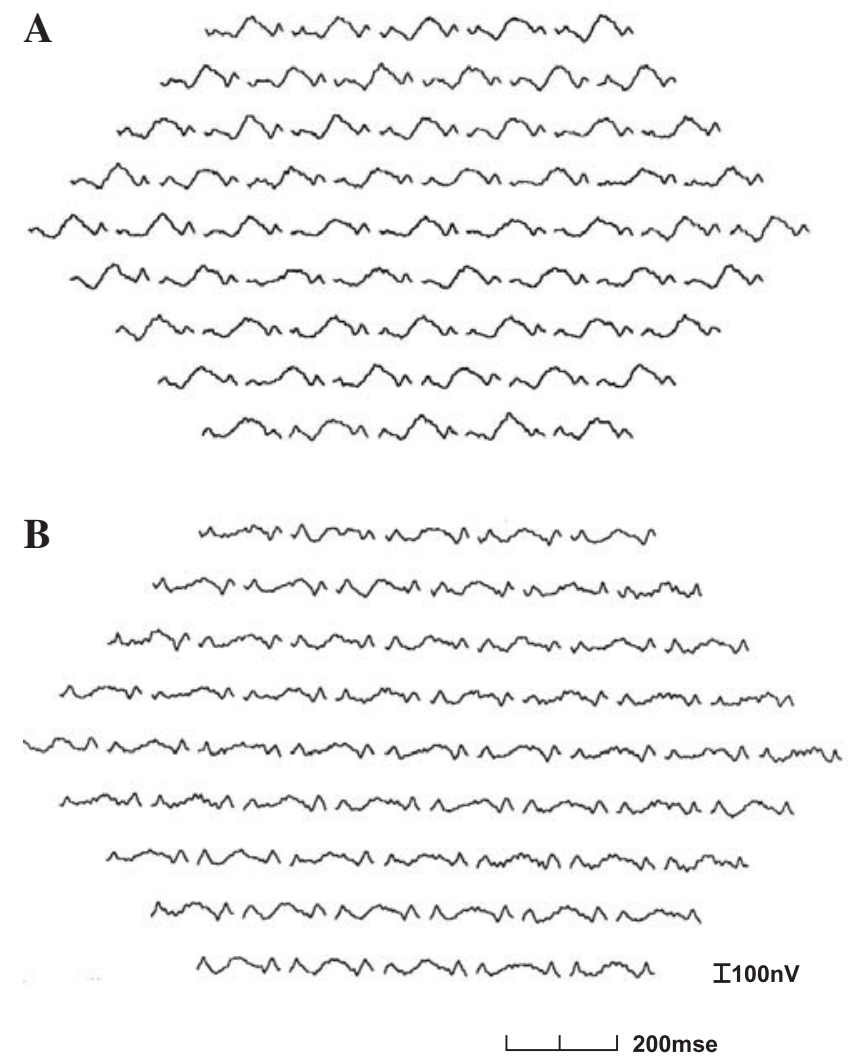

FIG. 3. Multifocal electroretinogram of the non-electroacupuncture (EA) treatment groups from the (A) left control eye and (B) the right glaucomatous eye of a rat in the non EA treatment group. The peaks of P1 were attenuated in the glaucomatous eye compared to those seen in the control eye. This illustrates that glaucomatous damage can affect the waveform characteristics.

$p>0.05 ; \mathrm{P} 1, t=-0.62, p>0.05)$. In addition, in the comparison of the $\mathrm{N} 1$ and $\mathrm{P} 1$ responses of the glaucomatous and control eyes, there were no differences among all three groups (N1 response in glaucomatous eye: non-EA versus $2 \mathrm{~Hz}$ EA, $t=1.77, p>0.05$; non-EA versus $100 \mathrm{~Hz}$ EA, $t=1.28, p>0.05$; P1 response in glaucomatous eye: nonEA versus $2 \mathrm{~Hz}$ EA, $t=0.76, p>0.05$; non-EA versus 100 $\mathrm{Hz}$ EA, $t=0.92, p>0.05$; N1 response in control eye: nonEA versus $2 \mathrm{~Hz}$ EA, $t=-0.65, p>0.05$; non-EA versus $100 \mathrm{~Hz}$ EA, $t=-0.76, p>0.05$; P1 response in control eye: non-EA versus $2 \mathrm{~Hz}$ EA, $t=0.18, p>0.05$; non-EA versus $100 \mathrm{~Hz} \mathrm{EA}, t=-0.73, p>0.05$ ). The glaucomatous eyes without any EA treatment had abnormal increases in N1 and P1 amplitude as compared to the control eyes although the comparison is not statistical significant. This illustrated that the glaucomatous damage causes the functional changes in the retina. For both EA treatment groups, the glaucomatous eyes did not show any abnormal increase in amplitude as compared to the control eyes, demonstrating the tentative effect of EA on glaucoma treatment. Therefore, we plan to change our future study design to investigate the effect of EA on glaucoma treatment. 


\section{mfERG response ratio (N/P ratio)}

Change of waveform characteristics is an important sign of glaucomatous damage in mfERG response. The N/P ratio from this study was analyzed to estimate retinal function. The N1 and P1 responses have opposite polarities and they influence each other in a manner similar to the a-wave and b-wave in flash ERG. In a normal flash ERG waveform under fixed measuring conditions (following ISCEV guidelines), the waveform characteristic is related to the a-wave to b-wave amplitude ratio. An abnormal ratio of a-wave to b-wave reflects abnormality in the retinal function. We postulate that the same concept is also true for the mfERG waveform.

Thus, N/P ratio waveform changes are analyzed to extrapolate the retinal function under different EA treatment conditions. In the treatment groups, the N/P ratios from all the control eyes showed similar values, but relatively larger differences were found in the glaucomatous eyes (Fig. 5).

In the non-EA control group, the N/P ratio of the glauco-

A

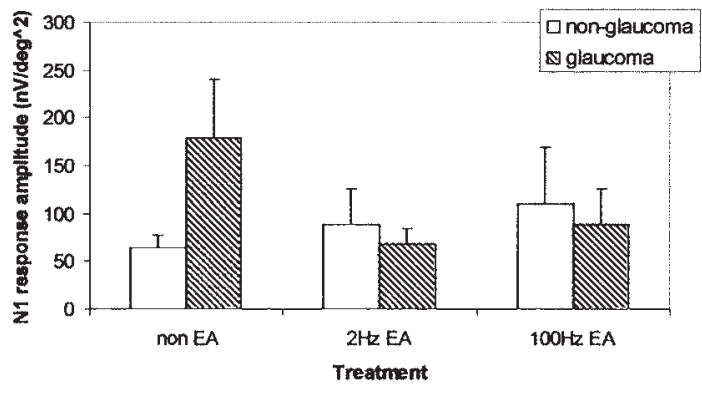

B

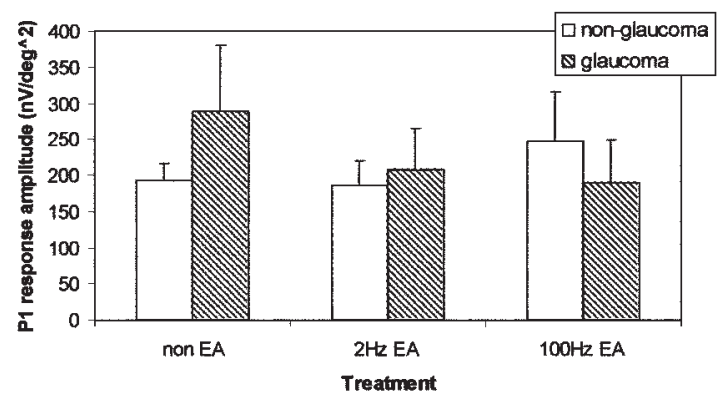

FIG. 4. Amplitudes (mean and SEM) of A. N1 and B. P1 in induced-glaucoma and control eyes. In the non-electroacupuncture (EA) group, there were large differences (50 to 64\%) in both $\mathrm{N} 1$ and P1 responses between the control eyes and the glaucomatous eyes. For the $2 \mathrm{~Hz}$ and $100 \mathrm{~Hz}$ EA groups, there was less difference (10 to 20\%) in the $\mathrm{N} 1$ and $\mathrm{P} 1$ responses between the control eyes and the glaucomatous eyes. These suggest that the retinal function of glaucoma eyes may have been preserved in both EA treatments. However, the high variances of the results renders the data statistically insignificant $(p>0.05)$ in all three groups.

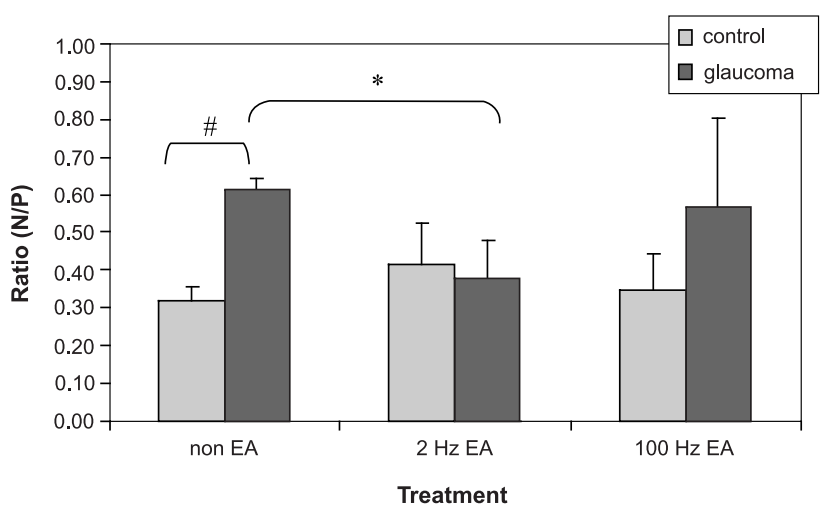

FIG. 5. N/P Ratios of the induced-glaucomatous and control eyes. In the non-electroacupuncture (EA) group, there was a large difference (about 50\%) between the control eyes and the glaucomatous eyes $(\# p<0.01)$. This illustrates that the glaucomatous eyes had significant changes in the N/P ratio. For the $2-\mathrm{Hz}$ and $100-\mathrm{Hz}$ EA groups, it was clearly illustrated that the 2-Hz EA group maintained the N/P ratio in glaucoma eyes $(p>0.05)$ but the $100-$ $\mathrm{Hz}$ EA group $(p>0.05)$ did not. In the comparison of the N/P ratio of glaucomatous eyes between the non-EA and $2 \mathrm{~Hz}$ EA groups, the difference was statistically significant $(* p<0.05)$, but this was not the case between the non-EA and the $100-\mathrm{Hz}$ EA groups ( $p>$ $0.05)$. In the comparison of the N/P ratio of control eyes, there were no statistically differences among all three treatment and control groups $(p>0.05)$.

matous eyes was significantly higher than that of the control eyes $(t=5.81, p<0.01)$. In the $2 \mathrm{~Hz}$ EA group, the mean $\mathrm{N} / \mathrm{P}$ ratio and standard deviation $(\mathrm{SD})$ of the glaucomatous eyes were similar to that of the control eyes and there was no significant difference between them $(t=-0.26, p>0.05)$. In the $100 \mathrm{~Hz}$ EA group, the mean N/P ratio of the glaucomatous eyes was higher than that of the control eyes. However, the difference was not significant due to the large SD of the ratios in the glaucomatous eyes $(t=0.87, p>0.05)$.

In the comparison of the N/P ratio of glaucomatous eyes between the non-EA and $2 \mathrm{~Hz}$ EA groups, there was a statistically significant difference $(t=2.18, p<0.05)$, but not between the non-EA and $100 \mathrm{~Hz}$ EA groups $(t=0.19, p>0.05)$. This demonstrated that $2 \mathrm{~Hz}$ EA treatment can influence the N/P ratio of the glaucoma eye, but not the $100 \mathrm{~Hz}$ EA treatment. In the comparison of the N/P ratio of control eyes among all three groups, there was no difference (non-EA versus $2 \mathrm{~Hz}$ EA, $t=-0.85, p>0.05$; non-EA versus $100 \mathrm{~Hz}$ EA: $t=$ $-0.26, p>0.05)$, that is, the EA treatment had no effect on the control eyes. The results show that the $2 \mathrm{~Hz}$ EA treatment can maintain the N/P ratio (ERG waveform characteristics) of the glaucomatous eye similar to that of the normal eye.

\section{DISCUSSION}

Previous studies have suggested that glaucomatous damage is a result of nitric oxide (NO) toxicity (Fisher et 
al., 2001; Morgan et al., 1999; Neufeld et al., 1999; Siu et al., 2002). Our group, using a similar rat glaucoma model, has found that acupuncture treatment could inhibit the expression of NOS-2 (Leung et al., 2000). In a recent study, we used acupuncture points based on the "local point" principal in another animal model (Siu et al., 2004), and found them effective. In the present study, using the same acupuncture points, we show that EA is effective in maintaining retinal function (i.e., mfERG) in the glaucoma rat model. Although the mfERG was not measured before the EA treatments, the control eyes were used for comparing the results, and the selection of glaucoma-induced rats for each treatment group was random to ensure that there was no bias to the different EA treatment conditions.

Laquis and colleagues (1988) reported that the percentage of RGC death was about $4 \%$ per week after induced IOP elevation whereas our laboratory has found that RGC death reached about $30 \%$ after 5 weeks of IOP elevation (Siu et al., 2002). Therefore, the estimated RGC loss would be $20-30 \%$ after 5 weeks of IOP elevation. Mittag and coworkers (2000) used the scotopic full-field ERG to investigate functional retinal changes in a glaucoma rat model and found that the decline in amplitude in the ERG responses of the glaucomatous eyes was not significant until after 15 weeks of elevated IOP. This illustrates that the amount of dead/damaged RGCs at 5 weeks of IOP elevation may not be high enough to express reductions in mfERG response.

The present study shows that there is only a moderate difference in the absolute mfERG amplitudes of N1 and P1 between the glaucomatous and control eyes in both treatment groups. Some studies have reported changes of waveform characteristics as a sign of glaucomatous damage in human mfERG response, even in the absence of any significant reduction in the response amplitude (Bearse et al., 1996; Hasegawa et al., 2000; Hood et al., 1999; 2002). Thus, the N/P ratio in waveform changes may be useful in the interpretation of the retinal function under different EA treatment conditions since the negative trough $(\mathrm{N})$ and the positive peak $(\mathrm{P})$ in the mfERG are related to the activities of off-bipolar and on-bipolar cells respectively (Hood et al., 2002). The glaucomatous damage may cause functional changes in these cells. Lau et al. (1994) have found that NOS is mainly secreted from the amacrine cells which are directly or indirectly connected to the bipolar cells. This can explain why the N/P ratio would reflect retinal function. The glaucomatous eyes receiving $2 \mathrm{~Hz}$ EA treatment showed N/P ratios similar to those of the control eyes. In a recent study, using mfERG measurement on monkeys with experimentally induced glaucoma and reared under low luminance or low contrast conditions, the N/P ratio was increased in the glaucomatous eyes (Raz et al., 2002). This supports the hypothesis that the N/P ratio is a possible indicator for measuring glaucomatous damage. Leung and co-workers (2000) have shown that the amount of NOS-2 is higher in glaucomatous eyes but is reduced after receiving $2 \mathrm{~Hz}$ EA treatment. In this study, the normal waveform characteristics were maintained in the glaucomatous eyes receiving EA treatment, which may be attributed to the inhibition of NOS-2. It is interesting to note that the neuroprotective effect of low frequency EA on the glaucomatous eye in the present study was also found in a cerebral ischemia model (Siu et al., 2004) On the other hand, the glaucomatous eyes receiving $100 \mathrm{~Hz}$ EA treatment did not show N/P ratio values similar to the control eyes. This might indicate that $2 \mathrm{~Hz}$ EA is effective in protecting the retina from insults, while $100 \mathrm{~Hz}$ EA produces little or no effect.

Comparing the effects of $2 \mathrm{~Hz}$ and $100 \mathrm{~Hz}$ EA treatments on retinal function in this study, the $2 \mathrm{~Hz}$ EA treatment preserved mfERG waveform characteristics in terms of the N/P ratio. Low and high stimulation frequencies of EA treatment seem to have different effects on the nervous system. In previous studies on the analgesic effect of EA, the $\beta$-endorphin release in rats was stimulated by $2 \mathrm{~Hz}$ EA but was not 100 Hz EA (Han et al., 1999; Huang et al., 2000).

We speculate that different EA treatment frequencies trigger different neuroprotective responses in the glaucomatous eye. Further investigation on the effects of low and high EA stimulation on NOS-2 in glaucoma is required.

\section{CONCLUSIONS}

This study demonstrates the neuroprotective effect of EA on glaucoma in rats by measuring their retinal responses. This study suggests that EA could be a potential treatment in preventing glaucomatous damage in human, but further investigation is necessary. Different EA stimulation frequencies produce different effects and low stimulation frequency $(2 \mathrm{~Hz})$ EA was preferable in treating glaucoma in rats. EA could be a good alternative therapy and novel approach to dealing with obstinate neurodegenerative diseases such as glaucoma. We hope that further research will be undertaken to explore this approach.

\section{ACKNOWLEDGMENTS}

This study was supported by Departmental Research Grant G-T012, Area of Strategic Development Research Grant A106, grant GT636 from The Hong Kong Polytechnic University, and a research grant from the University of Hong Kong. The authors thank Man-Chun Tsoi, B.Sc., LaiPing Wong, B.Sc., Sze-Man Yip, B.Sc., and Flora Ka-Wai Siu, Ph.D., for their help with the measurements. 


\section{REFERENCES}

Anderson DR. The optic nerve. In: Moses RA, Hart WM (eds.) Adler's Physiology of the Eye: Clinical Application, 8th ed. St. Louis: Mosby, 1987:497-501.

Bearse MAJ, Shutter EE, Sim D, Stamper R. Glaucomatous dysfunction revealed in higher order components of the electroretinogram. In: Vision Science and Its Application. Washington, DC: OSA Technical Digest Series, 1996:104-107.

Becquet F, Courtois Y, Goureau O. Nitric oxide in the eye: multifaceted roles and diverse outcomes. Surv Ophthalmol 1997;42: $71-82$.

Chan HL, Brown B. Multifocal ERG changes in glaucoma. Ophthal Physiol Opt 1999;19:306-316.

Chan HH, Brown B. Pilot study of the multifocal electroretinogram in ocular hypertension. Br J Ophthalmol 2000;84:11471153.

Dabov S, Goutoranov G, Ivanova R, Petkova N. Clinical application of acupuncture in ophthalmology. Acupunct Electrother Res 1985;10:79-93.

Dreyer EB, Zurakowski D, Schumer RA, Podos SM, Lipton SA. Elevated glutamate levels in the vitreous body of humans and monkeys with glaucoma. Arch Ophthalmol 1996;114:299-305.

Fisher J, Levkovitch-Verbin H, Schori H, Yoles E, Butovsky O, Kaye JF, Ben-Nun A, Schwartz M. Vaccination for neuroprotection in the mouse optic nerve: implications for optic neuropathies. J Neurosci 2001;21:136-142.

Garcia-Valenzuela E, Shareef S, Walsh J, Sharma SC. Programmed cell death of retinal ganglion cells during experimental glaucoma. Exp Eye Res 1995;61:33-44.

Han Z, Jiang YH, Wan Y, Chang JK, Han JS. Endomorphin-1 mediates $2 \mathrm{~Hz}$ but not $100 \mathrm{~Hz}$ electroacupuncture analgesia in the rat. Neurosci Lett 1999;22:75-78.

Hasegawa S, Takagi M, Usui T, Takada R, Abe H. Waveform changes of the first-order multifocal electroretinogram in patients with glaucoma. Invest Ophthalmol Vis Sci 2000;41:15971603.

Hood DC, Frishman LJ, Saszik S, Viswanathan S. Retinal origins of the primate multifocal ERG: Implications for the human response. Invest Ophthalmol Vis Sci 2002;43:1673-1685.

Hood DC. Assessing retinal function with the multifocal technique. Prog Retin Eye Res 2000;19:607-646.

Hood DC, Greenstein V, Frishman L, Holopigian K, Viswanathan S, Seiple W, Ahmed J, Robson JG. Identifying inner retinal contributions to the human multifocal ERG. Vision Res 1999;39: 2285-2291.

Huang C, Wang Y, Chang JK, Han JS. Endomorphin and $\mu$-opioid receptors in mouse brain mediate the analgesic effect induced by $2 \mathrm{~Hz}$ but not $100 \mathrm{~Hz}$ electroacupuncture stimualtion. Neurosci Lett 2000;294;159-162.

Ji JZ, Elyaman W, Yip HK, Lee VWH, Yick LW, Hugon J, So K.-F. CNTF promotes survival of retinal ganglion cells after induction of ocular hypertension in rats: the possible involvement of STAT3 Pathway. Eur J Neurosci 2004;19:265-272.

Kerrigan LA, Zack DJ, Quigley HA, Smith SD, Pease ME. TUNEL-positive ganglion cells in human primary open-angle glaucoma. Arch Ophthalmol 1997;115:1031-1035.

Laquis S, Chaudhary P, Sharma SC. The patterns of retinal ganglion cell death in hypertensive eyes. Brain Res 1998;16: 100-104.
Lau KC, So KF, Tay DKC, Leung MCP. NADPH-diaphorase neurons in the retina of the hamster. J Comp Neurol 1994;350: $550-558$.

Leung MCP, Chan HL, Butt YKC, Ji JZ, So KF. Electro-acupuncture decreases the activity and expression of nitric oxide synthase in a rat glaucoma model. Nitric Oxide Biol Chem 2000; $4: 288$.

Levkovitch-Verbin H, Quigley HA, Martin KR, Valenta D, Baumrind LA, Pease ME. Translimbal laser photocoagulation to the trabecular meshwork as a model of glaucoma in rats. Invest Ophthalmol Vis Sci 2002;43:402-410.

Lin B. Treatment of frontal headache with acupuncture on zhongwan-a report of 110 cases. J Tradit Chin Med 1991;11:7-8.

Lipton SA, Choi YB, Pan ZH, Lei SZ, Chen HS, Sucher NJ, Loscalzo J, Singel DJ, Stamler JS. A redox-based mechanism for the neuroprotective and neurodestructive effects of nitric oxide and related nitroso-compounds. Nature 1993;364:626-632.

Mittag TW, Danias J, Pohorenec G, Yuan HM, Burakgazi E, Chalmers-Redman R, Podos SM, Tatton WG. Retinal damage after 3 to 4 months of elevated intraocular pressure in a rat glaucoma model. Invest Ophthalmol Vis Sci 2000;41:3451-3459.

Morgan J, Caprioli J, Koseki Y. Nitric oxide mediates excitotoxic and anoxic damage in rat retinal ganglion cells cocultured with astroglia. Arch Ophthalmol 1999;117:1524-1529.

Neufeld AH, Sawada A, Becker B. Inhibition of nitric-oxide synthase 2 by aminoguanidine provides neuroprotection of retinal ganglion cells in a rat model of chronic glaucoma. Proc Natl Acad Sci U S A 1999;96:9944-9948.

Nickells RW. Retinal ganglion cell death in glaucoma: the how, the why, and the maybe. J Glaucoma 1996;5:345-356.

Nusinowitz S, Ridder III, WH, Heckenlively JR. Rod multifocal electroretinograms in mice. Invest Ophthalmol Vis Sci 1999;40: 2848-2858.

Quigley HA, Addicks EM, Green WR, Maumenee AE. Optic nerve damage in human glaucoma. II. The site of injury and susceptibility to damage. Arch Ophthalmol 1981;99:635-649.

Quigley HA, Nickells RW, Kerrigan LA, Pease ME, Thibault DJ, Zack DJ. Retinal ganglion cell death in experimental glaucoma and after axotomy occurs by apoptosis. Invest Ophthalmol Vis Sci 1995;36:774-786.

Raz D, Seeliger MW, Geva AB, Percicot CL, Lambrou GN, Ofri $\mathrm{R}$. The effect of contrast and luminance on mfERG responses in a monkey model of glaucoma. Invest Ophthalmol Vis Sci 2002;43:2027-2035.

Remtulla S, Hallett PE. A schematic eye for the mouse, and comparisons with the rat. Vision Res 1985;25:21-31.

Schori H, Kipnis J, Yoles E, WoldeMussie E, Ruiz G, Wheeler LA, Schwartz M. Vaccination for protection of retinal ganglion cells against death from glutamate cytotoxicity and ocular hypertension: Implications for glaucoma. Proc Natl Acad Sci U S A 2001;98:3398-3403.

Schwartz M, Belkin M, Yoles E, Solomon A. Potential treatment modalities for glaucomatous neuropathy: neuroprotection and neuroregeneration. J Glaucoma 1996;5:427-432.

Shao JM, Ding YD. Clinical observation on 111 cases of asthma treated by acupuncture and moxibustion. J Tradit Chin Med 1985;5:23-25.

Siu AW, Leung CP, To CH, Siu FKW, So KF. Total retinal nitric oxide production is increased in intraocular pressure-elevated rats. Exp Eye Res 2002;75:401-406. 
Siu FKW, Lo SCL, Leung MCP. Electroacupuncture reduces the extent of lipid peroxidation by increasing superoxide dismutase and glutathione peroxidase activities in ischemic-reperfused rat brains. Neurosci Lett 2004;354:158-162.

Song Z. Treatment of 1000 cases of lumbar soft tissue injury with acupuncture plus exercise. J Tradit Chin Med 1993;13:19-21.

Stux G, Pomeranz B. Basics of Acupuncture. Berlin: Springer-Verlag, 1988:1-42, 230-234.

WoldeMussie E, Ruiz G, Feldmann B. Effect of chronically elevated intraocular pressure on loss of retinal ganglion cells in rats. Invest Ophthalmol Vis Sci 1997;38:S159 [Abstract].

WoldeMussie E, Ruiz G, Wijono M, Wheeler LA. Neuroprotection of retinal ganglion cells by brimonidine in rats with laserinduced chronic ocular hypertension. Invest Ophthalmol Vis Sci 2001;42:2849-2855.
Yang R, Huang ZN, Cheng JS. Anticonvulsion effect of acupuncture might be related to the decrease of neuronal and inducible nitric oxide synthase. Acupunct Electrother Res 2000;25: 137-143.

Address reprint requests to: Henry H.L. Chan, Ph.D. Department of Optometry and Radiography Hong Kong Polytechnic University Hong Kong China

E-mail: orhenry@polyu.edu.hk 